\title{
L'approche générationnelle de la communication : placer les publics au cœur du processus
}

Myriam Bahuaud, Corinne Destal et Agnès Pecolo

\section{OpenEdition}

\section{Journals}

Édition électronique

URL : http://journals.openedition.org/communicationorganisation/3508

DOI : 10.4000/communicationorganisation.3508

ISSN : $1775-3546$

Éditeur

Presses universitaires de Bordeaux

Édition imprimée

Date de publication : 1 décembre 2011

Pagination : 5-18

ISBN : 978-2-86781-745-8

ISSN : $1168-5549$

Référence électronique

Myriam Bahuaud, Corinne Destal et Agnès Pecolo, « L'approche générationnelle de la communication : placer les publics au cœur du processus », Communication et organisation [En ligne], 40 | 2011, mis en ligne le 01 décembre 2014, consulté le 21 septembre 2020. URL : http://journals.openedition.org/ communicationorganisation/3508; DOI : https://doi.org/10.4000/communicationorganisation.3508 


\title{
L'approche générationnelle de la communication : placer les publics au cœur du processus
}

\author{
Mụriam Bahuaud, Corinne Destal et Agnès Pecolo
}

Lobjectif de ce numéro est d'ouvrir un champ de réflexion et d'analyse communicationnelle sur la question des publics de la communication, en mettant en lumière les représentations, discours et pratiques autour des nouvelles et anciennes générations, des jeunesses et vieillesses. Ce numéro propose donc un ensemble de recherches en Sciences de l'information et de la communication qui investit les problématiques de l'âge, des cycles de vie et des générations.

L'intérêt d'une approche générationnelle est qu'elle permet de saisir les tendances culturelles des différents groupes sociaux constituant la société, d'en comprendre les évolutions (les passages entre hier, aujourd'hui et demain) et de (ré)interroger les cohabitations entre les générations dans les différentes sphères publiques ou privées, familiales ou organisationnelles. En parallèle des segmentations communicationnelles par âges et cycles de vie, le marketing se fait transgénérationnel, certains médias se veulent intergénérationnels, l'entreprise se concentre plus sur la gestion des âges de la vie qu'à la politique d'un âge, les collectivités se polarisent sur les solidarités et cohésions sociales. La communication, quel que soit son champ d'application, s’interroge sur la segmentation ou non de ses publics, sur les représentations des âges et cycles de vie, sur les profils des générations en vie, autant d'éléments qui impactent sur ses stratégies et qui sont, dans ce numéro, questionnés.

\section{Approches générationnelles}

\section{Entre âges et générations, entre pairs et pères}

Aborder la question des pratiques de communication par le prisme générationnel, c'est rencontrer les notions d'âge (des cohortes démographiques nées à la même date) et de cycles de vie (enfance, jeunesse, vieillesse et les passages entre ces périodes). C'est aussi s'interroger sur la notion de génération. La génération renvoie à la fois à la succession dans le temps des individus (de pères en fils / idée de la filiation) quand, dans un même temps, une génération 
définit un groupe social constitué (entre pairs), plus ou moins artificiellement, et auquel on accole généralement un petit nom (Verdun, Gorby, Tanguy...) (Préel, 2005). Une génération se différencie de la génération précédente (construction en opposition) du fait de marqueurs culturels et idéologiques spécifiques; elle ne devient à ce titre une génération que si elle a conscience d'en être une (Attias-Donfut, 1988), même si les médias et le marketing usent et abusent des surnoms pour des individus, premiers étonnés, de se découvrir " génération Tanguy ", « Dolto » ou " Gloss ».

Dès lors, conjuguer la communication et les générations permet d'analyser des pratiques communicationnelles en croisant les impacts de l'âge et des profils générationnels (et non plus seulement selon des origines socioculturelles ou le genre). Par-delà toutes les différences (sexuelle, ethnique, sociale...) dont il s'agit bien entendu de ne pas nier l'existence pour autant, l'idée générationnelle (un ciment commun porté par un groupe d'âge qui a été jeune au même moment) amène des pistes d'analyse intéressantes.

Travailler sur des pratiques communicationnelles à la lumière des générations, c'est croiser une appréhension horizontale (la culture des pairs) et verticale (culture des pères) car on se construit toujours entre ses âges et héritages. Les mouvances communicationnelles gagnent à être analysées par le prisme d'une lecture générationnelle double, resituant l'individu dans son histoire personnelle et collective.

\section{Effets d'âge, d'époque et de génération : décrypter des " profils " d'humanité}

Face à un comportement, une attitude ou un usage constatés, l'approche générationnelle va chercher à mieux en cerner les origines, en se posant au moins deux questions : est-ce dû à « un effet d'âge » ou plus particulièrement à « un effet de génération » ? (Bahuaud et Pecolo, 2010).

L'effet d'âge présuppose que certaines pratiques peuvent être associées à des cycles de vie, susceptibles de disparaître ou d'apparaître avec l'âge. Une pratique que l'on quittera en grandissant ou adoptera en vieillissant. L' « effet générationnel » explique une attitude par des habitudes prises dans la jeunesse et qui perdureront en vieillissant. Puisqu'avoir eu 20 ans en 2000 ou en 1960 n'est pas neutre pour un individu qui sera marqué (marqueurs générationnels) par son contexte de vie, l'« effet d'époque » (l'« esprit du temps », les conjonctures et propositions d'une société) se doit d'être également analysé.

L'effet d'époque ne peut que s'imbriquer, car intimement lié, aux deux autres. Une société à un moment donné véhicule tout son lot de représentations, attitudes et comportements, fonctions et statut associés, aux divers âges de la vie. Ainsi, arriver à démêler ces impacts n'est pas sans enjeu dans la mesure où l'on peut, à travers ces analyses, lire un peu l'avenir et par conséquent établir des prospectives sur l'évolution des pratiques organisationnelles et communicationnelles, et notamment médiatiques (Donnat et Levy, 2007). Ceci étant, rien n'est moins délicat que de dresser des diagnostics définitifs 
dans la mesure où l'analyse de la plupart des pratiques communicationnelles reste complexe, du fait de la multiplicité des facteurs qui entrent en compte, à commencer par la réintroduction nécessaire des histoires de vie et tout ce qui fait qu'un individu reste unique.

\section{Approches intra, inter et transgénérationnelles}

\section{Des concepts pour recréer du lien social}

L'enchaînement des générations éclaire donc l'idée d'un enfantement. C'est une part de nous-mêmes que nous transmettons à nos descendances ; il n'y a pas eux et nous mais bien nous dans eux.

Les pratiques communicationnelles et médiatiques juvéniles ne sont plus stigmatisées comme exclusives et venues de nulle part. Nous pouvons grâce aux éclairages générationnels positionner la jeunesse d'après-guerre (leurs aînés) comme une digne représentante de la course à la consommation et retenir par ailleurs, avec intérêt, la thèse de la consommation " cicatrice » palliant la crise des référents, l'approche tribale de la marque en lieu et place des liens de parenté, la marchandise et les images comme substituts symboliques aux liens traditionnels (Heilbrunn, 2010). L'abandon du sens de la communauté intergénérationnelle permettrait aux marques de remplir une fonction identitaire compensatoire dans le cadre de la communauté des pairs. Les marques en prenant le relais des idéologies participent au « triomphe du marketing ». Le marketing, parce qu'il prend en compte l'opinion publique et vise à la convaincre, serait devenu un nouveau "métarécit » de nos sociétés occidentales (Firat et Venkatesh, 1993). Romain Laufer et Julien Lévy précisent : «Une société postmoderne est une société confrontée à une crise de la légitimité. L'opinion devient le juge final, le critère de légitimation principal. Le marketing, considéré comme une technique contemporaine de persuasion, peut ainsi être utilisé non seulement pour vendre des produits, mais aussi pour s'adresser à une variété de publics, sur lesquels les organisations reposent " (Laufer et Lévy, 1997). Ce processus se retrouve au sein de toutes les institutions, marchandes ou non, et modifie leur façon de fonctionner.

Le marketing est donc en filigrane sur l'ensemble du dossier (l'entreprise et son produit ou son histoire "à vendre ", les collectivités et leur intégration des logiques commerciales, les médias et leurs publics « cibles»). Parce que ce champ se concentre, entre autres, sur les publics, le concept de générations y est très présent, depuis les années 1980, aussi bien en recherche que sur un plan plus managérial (Bourcier-Béquaert et Barnier de, 2010). La communication générationnelle fait essentiellement référence à une problématique de segmentation et de ciblage, que ce soit pour privilégier une génération (la jeunesse étant certainement depuis les années 1960 la plus traditionnelle des " cibles ») ou pour, à l'inverse, essayer de rassembler plusieurs générations autour d'une marque. Cette segmentation communicationnelle favorise de 
fait une lecture générationnelle des pratiques, chaque individu étant supposé avoir des comportements sensiblement identiques du fait d'une année de naissance commune. Approche horizontale des générations, on appuie ici le concept de génération sociale (Mannheim, 2011).

Parallèlement la communication transgénérationnelle élabore un discours de façon à s'adresser à plusieurs générations à un même moment et devient, ainsi, capable de "traverser les générations » (Heilbrunn, 1999). Aussi, ce qui distingue le générationnel du transgénérationnel et le transgénérationnel du « tout public » est bien la notion de transversalité que nous retrouvons non plus uniquement de façon horizontale (autour de l'idée de rassemblement) mais également verticale (autour de l'idée d'enfantement, d'enchaînement dans le temps - générations familiales).

Croiser la communication et la notion de générations ce n'est donc pas se contenter de découper la population en générations (pour les opposer ou les distinguer), c'est se centrer sur ce qui peut, d'un point de vue communicationnel, recréer du lien social. C'est donc jouer la carte de la communication intergénérationnelle ou transgénérationnelle plutôt que générationnelle. L'intergénérationnel, concept présent, depuis la fin des années 1980 dans les colloques sur le grand âge (Krémer, 2001), et depuis les années 1990 dans les discours politique et social, « renvoie donc d'emblée à autrui, à la rencontre avec l'autre, plus jeune ou plus vieux ; il fait de chacun de nous un héritier ou un passeur de la mémoire, de l'histoire, des savoirs ou encore du patrimoine " (Quéniart et Hurtubise, 2009 ; p. 8). Car on ne saurait évacuer trop rapidement l'impact de la filiation et des héritages dans le champ communicationnel ; l'intergénérationnel transparaît dans les travaux sur les influences des parents sur les enfants, réciproques et également « inversées » des enfants sur les parents (Gollety, 2007). L'intergénérationnel nourrit encore les travaux autour des seniors (Chevalier, 1999), ceux autour de la question des transmissions entre générations et enfin ceux privilégiant l'entrée « famille».

\section{Une combinaison de discours intra et intergénérationnel}

Dans ce numéro, Raphaëlle Camous éclaire, en tant que spécialiste du marketing et de la communication auprès des " $0-25$ ans ", une approche double qui consiste plutôt à combiner, dans le champ de la prévention des risques, un positionnement intragénérationnel classique avec un discours qu'elle qualifie d'intergénérationnel pour toucher les adolescents. S'il lui semble très pertinent pour les marques de développer des campagnes exclusivement dédiées aux adolescents satisfaisant un objectif de différenciation de leurs parents, l'enjeu est différent dès lors que la prévention des risques est en cause. Si les spécialistes de la communication savent combien les seniors répugnent à être ghettoïsés et se confrontent à une gestion des âges, complexe, on oublie souvent que les adolescents rejettent toute stigmatisation de leur mode de 
vie, de leurs comportements et ont beaucoup de mal à accepter un discours moralisateur. Un message de prévention qui les vise explicitement prend toujours le risque d'être refusé. L'auteur, après avoir montré en quoi la prise de risque joue un rôle dans la construction identitaire des adolescents, admet l'intérêt de construire un discours spécifique, prenant en compte la perception particulière que les adolescents ont du danger (insister sur la perte de contrôle de soi et sur les conséquences concrètes et immédiates de la consommation). Elle conseille, par la suite, une entrée beaucoup plus générale qui vise à replacer l'adolescent au milieu de ses aînés (en posant ces derniers comme également concernés par une mise en danger de soi), évitant ainsi la stigmatisation de comportements « juvéniles».

\section{Quand les entreprises racontent leur histoire}

\section{L'entreprise communique sur son passé}

Si les transmissions familiales et scolaires occupent traditionnellement le devant de la scène et que les entreprises se saisissent sérieusement de la question face aux problématiques de renouvellement générationnel (départ des baby-boomers et arrivée des $\mathrm{Y}^{1}$ ), il est moins courant de s'interroger, comme le propose ici Isabelle Cousserand, sur ces récits qui circulent au sein des entreprises, générations après générations, et dont elle déchiffre les constantes. Les « sagas » d'entreprises peuvent s'entourer d'une dimension patrimoniale mais aussi intemporelle et immémoriale permettant de tricoter une sorte de mythe auquel sont censées adhérer des générations de salariés et de consommateurs. Autre démarche, autre dimension, celle d'innovation et de challenge plus particulièrement sur le plan technique, qui appelle cette fois-ci la projection dans le futur. L'entreprise se pose comme en perpétuel perfectionnement et garante d'un avenir prometteur, un symbole pour les générations futures. "Susciter de l'attente et de la mémoire ", vanter savoir ancestral et prises de risques, tels sont les modes utilisés par ces récits d'entreprises qui veillent par ailleurs à procurer du « mieux vivre » et à s'ériger, forte de leur succès, comme référence. À travers cette stéréotypie des sagas, ce sont des processus identificatoires à travers le passé que l'on visite, cette manière qu'ont les entreprises de raconter leur histoire, une forme d'esthétisation de l'entreprise à travers son passé. Au service d'une dynamique de cohésion, d'authenticité et de destin commun, les récits tissent pour l'entreprise un continuum transgénérationnel où les clivages générationnels n'ont pas leur place.

1. On appelle génération Y les jeunes nés après 1980, soit généralement les 18-30 ans. On trouve à l'origine de cette dénomination la typologie des sociologues américains Neil Howe et William Strauss (1991). 


\section{Les communications nostalgiques}

C'est une autre forme de récit dans lequel Daphné Duvernay nous plonge en explorant, à travers l'exemple du marketing de Danette, ce que l'on nomme le marketing de la nostalgie (Badot et Cova, 2003 ; Divard et Robert-Demontrond, 1997) afin de pouvoir séduire les anciens enfants devenus adultes nostalgiques et les nouveaux enfants que l'on souhaite curieux d'hier. L'auteur en analysant l'évolution de la communication médiatique de Danette pointe plusieurs glissements parallèles. Celui d'une verticalité des pères à une horizontalité des pairs. C'est également le glissement d'un cycle de vie produit à un cycle de vie client. Les mouvances du marketing dans une société de plus en plus individualisée, mais aussi participative, sont en effet palpables. Ainsi Danette, s'appuyant sur une démarche de co-création collaborative, se focalise sur des opérations participatives sur le Net à l'occasion des 40 ans de la marque. Mais cette utilisation des réseaux comme support de mobilisation et de communication virale finit par brouiller, selon l'auteur, la stratégie de segmentation des cibles visées par la marque. Tout se passe comme si Danette oscillait entre un positionnement transgénérationnel fédérateur (la Danette produit de toutes les générations) et une segmentation centrée principalement sur les générations X (nostalgie de leur enfance sous l'ère Danette) et $\mathrm{Y}$ sensibles au rétromarketing en recourant aux réseaux socionumériques, réputés " jeunes ». Et les figures des grands-parents présents du temps de la communication intergénérationnelle, de disparaître de la scène...

\section{L'entreprise, ses jeunes et ses vieux}

\section{Génération Y, l'arrivée des juniors en entreprise}

Arrivée à l'âge adulte de la première génération née avec les TIC qu'elle maîtrise mais aussi fille des mutations socio-économiques amorcées avant sa naissance, la génération Y fait couler beaucoup d'encre, notamment depuis son arrivée sur le marché de l'emploi (Dagnaud, 2011 ; Levain et Tessier, 2012 ; Pouget, 2010). Génération ultra connectée (d'où le qualificatif de « digital natives $»^{2}$ ), elle s'empare du clavier pour changer le monde et invente de nouveaux modes de vie. Son mode de socialisation, différent de celui de ses aînés, entraine une rupture culturelle dans le sens où les référentiels mutent notamment dans le rapport au travail et plus loin dans le rapport aux systèmes et structures organisationnels hérités des ainés (Méda et Vendramin, 2010 ; Delay, 2008).

Coopération ou conflit, respect ou indifférence, les pratiques entr'âges et générations au sein des organisations ouvrent un vaste champ d'interrogations

2. Notion associée à Marc Prensky (2001) pour caractériser le comportement d'individus nés avec le numérique. 
(Guillemard, 2010 ; Marbot, 2007 ; Lambert, 2009 ; Lahouze-humbert, 2010).

Dans ce numéro, Namoin Yao propose une réflexion autour de la communication de recrutement. À travers l'image travaillée de l'entreprise pour recruter, est exposée une approche qu'elle nomme "clientéliste " (considérer les collaborateurs, présents ou potentiels, comme des clients). Plus précisément, parce que les organisations étudiées s'adressent prioritairement aux jeunes diplômés issus de la génération dite "Y ", l'article analyse ce positionnement générationnel. Grâce à une enquête qualitative auprès de responsables des ressources humaines, cette contribution met en évidence la manière dont leurs représentations des jeunes diplômés influencent leurs stratégies communicationnelles, notamment par la mobilisation d'outils de recrutement censés correspondre à cette cible : utilisation de Facebook, salons virtuels sur Second Life et autres speed recruiting.

Retenons que les politiques de gestion des ressources humaines se confrontent clairement aujourd'hui à la pyramide des âges pour gérer le renouvellement de leurs effectifs et que les politiques de l'emploi se penchent en urgence sur la catégorie " travailleur âgé ».

\section{Discrimination par l'âge : au-delà des seniors}

$\mathrm{Au}$-delà d'une perception toute personnelle de son âge et de celui de son voisin (on est tous le vieux ou le jeune de quelqu'un), on trouve autant de relativité dans la définition de la "personne âgée » que dans celle de la « jeune personne ». La vieillesse, tout comme la jeunesse, n'est bien évidemment qu'un mot (l'hétérogénéité prime, les avenirs et moyens, enjeux et milieux varient) et les représentations sociales des âges vont avec des sortes d'us et coutumes que l'on attribue hâtivement à chaque catégorie (Pecolo, 2009).

La lutte contre les discriminations par l'âge ${ }^{3}$, dont la manifestation la plus courante est attachée à la problématique de l'emploi et des seniors, s'étend pourtant au-delà du monde de l'entreprise et concerne tous les âges, pour devenir un combat contre l'âgisme. Les jeunes relèvent a priori moins de ce type de discrimination. Pourtant, ils font l'objet de mesures liées à l'âge qui peuvent être entendues comme des prescriptions excluantes (interdiction de consommation d'alcool avant 16 ans, RSA jeune créé en 2010 pour les 18-25 ans). La lutte contre la mise en place de barrières d'âge doit jongler entre la non-exclusion et des impératifs de protection (aux confins de l'existence, les vulnérabilités des jeunes et des personnes âgées imposent des mesures de protection particulières, juridiques et sociales notamment). On peut mettre en garde contre une société neutre du point de vue de l'âge qui exigerait alors de reconfigurer sa protection sociale (Caradec, Poli, Lefrançois, 2009).

3. L'âge fait maintenant partie des critères de discrimination surveillés par la HALDE (Haute autorité de lutte contre les discriminations et pour l'égalité). 
La discrimination par l'âge, en-dehors de ce cadre légal spécifique, est portée par les préjugés de tout un chacun. Elle émane des histoires vécues ou fantasmées, des croyances sociales, des représentations médiatiques.

C'est dans le monde du travail, espace où se cristallise le plus souvent cette discrimination, que s'installe l'étude de Nathalie Burnay. En s'arrêtant sur la cohabitation intergénérationnelle dans le contexte belge, l'auteur montre, grâce à une enquête auprès de 812 travailleurs de douze secteurs d'activité différents, combien les stéréotypes négatifs à l'encontre des travailleurs âgés hantent le monde du travail. Les plus jeunes générations et le secteur des services apparaissent comme les plus virulents. Les difficultés physiques ou d'apprentissage, cognitives ou mentales, la résistance au changement sont régulièrement évoquées quand les personnes plus âgées et moins qualifiées du secteur industriel appuient la richesse que représente l'expérience des seniors. Dans son article, l'auteur défend des changements organisationnels profonds pour lutter contre une discrimination liée à l'âge (directe ou indirecte), accompagnés d'un travail de communication visant à démystifier les soi-disant carences du senior. Transformer une logique transversale en une logique longitudinale pour la construction des carrières, des parcours de vie et des trajectoires professionnelles, permettrait une gestion des âges prévisionnelle soucieuse de développement des compétences et des ressources humaines.

\section{Médias et construction des publics}

\section{Médias et âgisme}

La discrimination par l'âge se manifeste également dans les représentations médiatiques. Souvent complexes et ambivalentes, elles dessinent les contours des espaces (de libertés et de restrictions) dans lesquels la vieillesse doit s'installer, et fournissent un terreau fertile à l'âgisme.

Martine Lagacé, Joëlle Laplante et André Davignon reviennent plus précisément sur le concept d'âgisme dont ils ne visitent pas que les manifestations explicites mais plutôt implicites et ce, dans le cadre d'une analyse médiatique d'écrits canadiens. Il est certain que les représentations médiatiques contribuent à la construction sociale des âges, véhiculent une perception de l'enfance ou de la vieillesse, tout comme ils accompagnent la construction identitaire tout au long de l'avancée en âge, en diffusant des modèles de conduite et des signes distinctifs. Les médias participent à une lecture des âges différentiée et les auteurs s'attachent ici à percer les images véhiculées de la vieillesse, du processus du vieillissement et des personnes âgées. Au-delà de la façon explicitement négative dont les médias dépeignent ces notions, contribuant ainsi à la cristallisation de croyances et d'attitudes âgistes, il ressort de leur analyse l'existence d'un discours médiatique âgiste polarisé. D'un côté la question du vieillissement traitée comme un fardeau 
social et économique à gérer, de l'autre celle des aînés qui parviennent à répondre aux normes de la jeunesse, dépeints de manière " positive » et alors encensés dans le discours médiatique. Ces stéréotypes contribuent à polariser les générations d'aînés entre elles et de ce fait, à nourrir une forme d'âgisme non plus seulement inter mais intra générationnelle.

Il est vrai que l'on peut couramment voir un découpage dans la catégorie « vieux » entre deux mondes : les « jeunes » seniors actifs et sportifs, en bonne santé et plein d'avenir (argentés et libérés des contraintes) et le grand âge synonyme quant à lui de dépendance et maladie. Perspectives économiques et culturelles d'un côté (Bahuaud, 2009), médicales et sociales de l'autre (Le $\mathrm{Ru}, 2008$; Carbonnelle, 2010). Cette opposition entre le «bien vieillir » et le « vieillir misérablement " malmène de façon violente les femmes âgées, victimes plus que d'autres, des regards sociaux déformants produits par une société dans laquelle le " paraître » et le « faire » importent.

Après avoir visité cette construction médiatique de représentations des âges et cycles de vie, c'est celle des publics effectifs que nous creusons selon deux niveaux : les renouvellements générationnels qui appellent des renouvellements culturels et la question du mélange des âges dans les fréquentations médiatiques en lien avec des stratégies communicationnelles.

\section{Médias et renouvellement générationnel}

La pratique des jeux vidéo ou une culture de séries, portées au départ et exclusivement par les jeunes des années 1980 ne semblent pas devoir être analysées comme un simple effet d'âge mais bien plus comme un effet générationnel qui implique notamment que les légitimités prêtées à ces supports et produits culturels ont évolué pour cette génération devenue adulte et parents (Pecolo, 2009). En effet, les premiers joueurs ayant grandi, on est en droit de se demander si la pratique des jeux vidéo est encore réservée aux adolescents (effet d'âge), ou si elle s'est généralisée à l'ensemble de la population du fait d'un effet générationnel stipulant que l'on grandit en conservant ses habitudes notamment médiatiques.

Typique de ce positionnement, le jeu vidéo fut très vite associé au jeune public plus spécifiquement de sexe masculin. Laurent Tremel aborde, ici, la question sous un angle intéressant en articulant la réalité d'un public « cible ", intéressé par des logiciels dont l'essence serait la " masculinité militarisée » (Kline, Dyer-Witheford, De Peuter, 2003) et la construction d'un public « voulu " par les industriels pour atteindre de nouvelles « cibles » (femmes, cadres, retraités, etc.). Et ce, par la mise en avant de «bons produits »(dont la promotion des serious games) s'éloignant ainsi des stéréotypes négatifs associés aux jeux vidéo (violence, machisme, néolibéralisme) et visant la modification de leur image sociale. Cette fabrique de l'opinion et par là d'un public au final fictif, ou pour le moins construit, permet de saisir l'impact des logiques commerciales souterraines. L'auteur, se situant clairement dans une 
perspective de sociologie critique, souhaite montrer comment les industriels peuvent instiller un discours sur les publics joueurs. Pour Laurent Tremel il ne fait aucun doute que la jeunesse masculine est prioritairement visée, ce que montre l'étude de l'ego sollicité dans les scénarios qui offrent une compensation symbolique et virtuelle à un réel juvénile moins rose (incertitude et précarité), grâce à l'incarnation par le joueur d'un avatar au destin hors du commun. Les chiffres publiés ces derniers temps sur une moyenne d'âge des joueurs comprise entre 30 et 39 ans est pour lui un pur artefact statistique, qu'il démonte dans son article, au service d'industriels en recherche tout à la fois de légitimité culturelle (détacher l'image du joueur d'un adolescent accro à des contenus violents) et de nouveaux marchés.

\section{Entre " pour " et " tout " public, entre " grand " et " petit " public}

Un média « pour» exploite des tendances socioculturelles et les conforte en contribuant à la diffusion (de par l'existence même du média) de l'idée qu'il puisse y avoir des besoins et attentes spécifiques... Segmenter c'est toujours prendre le risque de figer des castes d'âges en confortant des langages, valeurs et cultures spécifiques. Les contenus sont alors des miroirs proposés de représentations médiatiques d'eux-mêmes. Ils contribuent à la construction sociale des âges, véhiculent une certaine idée de l'enfance ou de la vieillesse. L'articulation segmentations économiques / ségrégations culturelles n'est jamais anodine et amène au-devant de la scène les questions du vivre ensemble et des solidarités tant publiques que privées. Les ségrégations générationnelles au même titre que celles urbaines et scolaires, raciales et sexuelles, sapent l'idée anthropologiquement défendue de favoriser l'Intergénérationnel, qu'approcher la culture de l'autre permet de réduire les distances qui effraient et offusquent.

Laurence Corroy nous présente l'étude d'un produit culturel susceptible de plaire non à l'un ou à l'autre mais à tous les âges de la vie. En nous emmenant au pays de "Plus belle la vie ", elle décrypte une offre conçue de manière générationnelle qui rencontre un fort succès sur France 3. Mettant en scène plusieurs générations, la série choisit la cohabitation de regards et de perceptions différentes sur une même action et favorise la confrontation de paroles qui n'est pas forcément synonyme de conflit culturel. On joue ici sur la complicité et la complémentarité générationnelles et non sur des rapports d'opposition et de "guerre des générations ». Ce sont même des parallèles générationnels que l'on peut évoquer lorsque l'auteur montre comment des thématiques transversales telles que les relations sentimentales ou la prévention dans le champ de la santé, sont abordées entre les plus âgés et les plus jeunes. Ce sont en effet les générations les plus extrêmes (grands-parents et petits enfants) qui sont particulièrement mises en scène (rôles centraux et valorisés) au fil des épisodes et non les quarantenaires présentés comme "en crise " (souvent plongés dans un désarroi parental, sentimental et/ou professionnel). 
« Plus belle la vie » surfe ainsi selon l'auteur sur un effet d'époque visant à valoriser les relations intergénérationnelles dans les nouvelles configurations familiales. Il est donc logique que l'on dresse un portrait apaisé des relations intergénérationnelles fait de connivence et intercompréhension. Le schéma de transmission véhiculé et analysé par Laurence Corroy comporte trois modalités (horizontale, verticale descendante et verticale ascendante), auxquelles elle associe pour chacune un registre de valeur (solidarité, cohésion sociale, modernité). Si l'on s'attarde sur le mode vertical ascendant de transmission (allant des juniors vers les seniors), nous ne serons nullement étonnés de trouver, comme savoirs associés, la maîtrise des TIC...

\section{Une exploration des territoires : cycles de vie et communication publique}

La petite enfance et l'enfance, l'adolescence et la jeunesse, les $3^{\text {ème }}$ et $4^{\text {ème }}$ âge mobilisent la sphère publique. Face au défi du vieillissement de la population, émergent de nouvelles interrogations sur les solidarités publiques et les cohésions sociales et donc des initiatives intergénérationnelles. Nous avons souhaité éclairer ce champ en donnant la parole à trois professionnels de la communication publique. Leur regard complète ce dossier et permet de constater comment les collectivités distinguent les cycles de vie dans leur communication, les découpages des populations classiquement opérés, les supports privilégiés en direction de chaque public générationnel et les représentations des âges sous-jacentes. Si les entrées famille, enfance et jeunesse sont désormais classiques, c'est l'émergence de celle de "seniors " et d'intergénérationnel que nous interrogeons avec eux plus précisément. Julien Goupil, fondateur et directeur du cabinet Proxité (agence spécialisée en communication sociale, publique et politique) nous précise les conditions qui ont favorisé l'émergence d'une logique de segmentation des publics et les spécificités de la communication territoriale dans le cadre d'une approche catégorisée des citoyens. Son expertise nous permet aussi d'explorer les supports et tons de communication favorisés en fonction des catégories d'âge mais également les entrées générationnelles les plus sollicitées par les collectivités. Enfin, c'est son approche du « vivre ensemble » dans sa traduction communicationnelle que nous recueillons. La perspective intergénérationnelle semble être une entrée en communication favorisée par les territoires au Québec. Didier Rigaud, en tant que spécialiste de la communication territoriale et collaborateur depuis plus de 20 ans de l'Association des communicateurs municipaux du Québec, nous propose ici une analyse comparative interculturelle. Nous terminons cette exploration en terrain politique avec une étude de cas centrée sur le positionnement communicationnel de la ville de Bordeaux, présentée au sein du réseau de la communication publique et territoriale (Cap'com) comme une des pionnières en communication seniors. Une politique du « bien vieillir » à promouvoir, une stratégie de communication plutôt transversale que réservée, de "vrais » 
seniors à représenter, telles sont, en résumé, les optiques que la direction de la communication souhaite véhiculer. En clair : "Oser les cheveux blancs ».

\section{BIBLIOGRAPHIE}

ATTIAS-DONFUT C., Sociologie des générations : l'empreinte du temps, Paris, PUF, «Le sociologue », 1988, 249 p.

BADOT O., COVA B., "Néo-marketing, 10 ans après : pour une théorie critique de la consommation et du marketing réenchanté ", Revue française du Marketing, n¹95, 2003, p. 79-94.

BAHUAUD M., PECOLO A., " De la segmentation à la confusion des âges : stratégies marketing et approches générationnelles des publics », Actes du colloque "Culture et identités dans les communications marketing " 78 e congrès de l'ACFAS, Centre d'études en communications marketing - UQAM, Université de Montréal, 11 mai 2010, p. 74-82.

BAHUAUD M., " Presse professionnelle en communication : le filon de l'or gris », Médias, cabier Médiamorphoses, 2009, n²0, p. 116-119.

BOURCIER-BÉQUAERT B., BARNIER V. de, « Pour un cadre élargi du concept de génération en marketing ", Recherche et Applications en Marketing, 2010, 25, n³, p. 115-135.

CARADEC V., POLI A. et LEFRANÇOIS C., « Les deux visages de la lutte contre la discrimination par l'âge ", La tyrannie de l'âge, 2009, 59, p. 12-23.

CARBONNELLE S. (dir.), Penser les vieillesses: Regards sociologiques et anthropologiques sur l'avancée en âge, Paris, Seli Arslan, 2010, 254 p.

CHEVALIER C., « La perception des publicités par les seniors », Décisions Marketing, 1999, n¹8, p. 41-49.

DAGNAUD M., Génération Y - Les jeunes et les réseaux sociaux, de la dérision à la subversion, Paris, Sciences Po (Les Presses de), « Nouveaux débats », 2011, 172 p.

DELAY B., Les rapports entre jeunes et anciens dans les grandes entreprises. La responsabilité organisationnelle dans la construction de dynamiques intergénérationnelles coopératives, Paris, Centre d'études de l'emploi, «Document de travail », 103, septembre 2008, 35 p.

DIVARD R., ROBERT-DEMONTROND P., « La nostalgie : un thème récent dans la recherche marketing ", Recherche et Applications en Marketing, 12, n4, p. 41-61.

DONNAT O., LÉVY F., "Approche générationnelle des pratiques culturelles et médiatiques ", Rapport du Département des études, de la prospective et des statistiques. Culture et prospective, Pratiques et publics, 2007, $32 \mathrm{p}$.

FIRAT F., VENKATESH A., "Postmodernism : the age of marketing », International Journal of Research in Marketing, 1993, 10, p. 227-249.

GOLLETY M., " La famille, un lieu d'échanges privilégiés pour apprendre à consommer », dans J. BRÉE (dir.), Kids marketing, Paris, EMS, 2007, p. 109-136. 
GUILLEMARD, A.-M., Les défis du vieillissement. Âge, emploi, retraite : perspectives internationales, Paris, Armand Colin, « Collection U Sociologie », 2010, 358 p.

HEILBRUNN B., « Les marques transgénérationnelles », Décisions marketing, 1999, $\mathrm{n}^{\circ} 18$, p. 81-84.

HEILBRUNN B., La consommation et ses sociologies, Paris, Armand Colin, «Domaines et approches », 2010, $125 \mathrm{p}$.

KLINE S., DYER-WITHEFORD N. et DE PEUTER G., Digital Play, Montréal \& Kingston, McGill-Queen's University Press, 2003.

KREMER P., «Des crèches et des maisons de retraite bousculent les frontières entre générations - L' "intergénérationnel", du discours à la réalité », Le Monde, 25 janvier 2001.

LAGACÉ M., L'âgisme : comprendre et changer le regard social sur le vieillissement, Québec, Presses de 1'Université Laval, 2010, 278 p.

LAHOUZE-HUMBERT É., Le choc générationnel : faire travailler ensemble trois générations, Paris, Maxima, 2010, 239 p.

LAMBERT J., Management intergénérationnel, Reuil-Malmaison, Éditions Lamarre, «Fonction cadre de santé. Gestion des ressources humaines », 2009, 269 p.

LAUFER R., LÉVY J., « «Postmodern Marketing» as a Pleonasm and «Liberatory Marketing» as Improbable - A Contribution to Marketing Theory and to the Debate on Marketing and Postmodernism ", Actes de la 1977 Winter Educators Conference, American Marketing Association, Tampa, 1997.

LE RU V., La vieillesse : de quoi avons-nous peur?, Paris, Larousse, " Philosopher ", 2008, 219 p.

LEVAIN M., TISSIER J., La génération Y par elle-même : quand les 18-30 ans réinventent la vie, Paris, Bourin éditeur, 2012, $201 \mathrm{p}$.

MANNHEIM K., Le problème des générations, Paris, Armand Colin, 2011, 162 p.

MARBOT É., "La gestion des âges et l'équité entre les générations », Retraite et société, 2007/2, n51, p. 103-125.

MÉDA D., VENDRAMIN P., « Les générations entretiennent-elles un rapport différent au travail ? ", SociologieS [En ligne], Théories et recherches, mis en ligne le 27 décembre 2010. Disponibilité et accès http://sociologies.revues.org/3349.

PECOLO A., «Ages, générations et segmentations », Médias, cahier Médiamorphoses, 2009, n $^{\circ} 22$, p. 87-91.

PECOLO A., "Médias et générations : irruption de la figure du senior ", Médias, cabier Médiamorphoses, 2009, n²0, p. 110-112.

POUGET J., Intégrer et manager la génération $Y$ : attirer cette nouvelle population active, riche d'opportunités, Paris, Vuibert, "Lire Agir. Ressources humaines », 2010, 203 p.

PRÉEL B., Les générations mutantes : Belle époque, krach, mai 68, Internet, quatre générations dans l'histoire, Paris, La Découverte, « cahiers libres », 2005, 283 p.

PRENSKY M., Digital Natives, Digital Immigrants, Site de Marc Prensky, [En ligne, page consultée le 28 février 2012]. Disponibilité et accès http://www.marcprensky. com/writing/prensky\%20-\%20digital\%20natives,\%20digital\%20immigrants\%20-\%20 part1.pdf. 
QUÉNIART A., HURTUBISE R. (dir.), L'intergénérationnel. Regards pluridisciplinaires, Rennes, Presses de l'EHESP, 2009, 299 p.

STRAUSS W., HOWE N. , Generations. The History of America's Future, New-York, Quill William Morrow, 1991. 